\title{
Phylenzahl und Phylenzyklus in Hermupolis und Oxyrhynchos
}

\author{
Holger Essler*
}

\begin{abstract}
While the tribal cycles in Oxyrhynchos are fairly well attested from AD 206 until 271, no system has been proposed for other periods or other metropoleis. On the basis of recently published texts a first attempt is made to reconstruct the tribal cycles in Oxyrhynchus and Hermopolis for the later part of the $4^{\text {th }}$ century.
\end{abstract}

Keywords: Hermopolis, Oxyrhynchos, tribes, liturgy, administration

DOI 10.1515/apf-2016-0011

Die Organisation der Bürgerschaft in Phylen gilt als traditionelles Kennzeichen einer hellenistischen Polis, ${ }^{1}$ das als Symbol der Zusammengehörigkeit und Grundlage der Selbstverwaltung besondere Wertschätzung geniesst. Waren in Ägypten Phylen zunächst nur für die griechischen Poleis Naukratis, Alexandria, Ptolemais und dann Antinoopolis bezeugt, erhalten unter Septimius Severus auch die Gaumetropolen eine Bule und eine Phylenordnung. Im Gegensatz zu den Phylennamen in den griechischen Poleis Ägyptens, die sich an Herrschernamen orientierten, wurden die Phylen in den Gaumetropolen entweder schlicht durchnumeriert oder nach den Stadtvierteln benannt, in denen ihre Mitglieder wohnten.

Eine der Hauptaufgaben der neu eingerichteten Phylen lag offensichtlich in der Organisation der von den Bürgern zu leistenden Liturgien, die in der Übernahme unterschiedlicher staatlicher Aufgaben in der öffentlichen Verwaltung, in der Steuererhebung, im Transportwesen, beim Un-

Vorbemerkung: Für Hinweise und Korrekturen danke ich A. Benaissa, D. Colomo, N. Gonis und D. Hagedorn.

* Kontakt: Holger Essler, Julius-Maximilians-Universität Würzburg, Institut für Klassische Philologie I, Residenzplatz 2, D-97070 Würzburg, <Holger.Essler@uni-wuerzburg. de>

${ }^{1}$ Die allgemeinen Ausführungen stützen sich auf Ursula Kunnert, Bürger unter sich. Phylen in den Städten des kaiserzeitlichen Ostens, Basel 2012, S. 19, 192-216. 
terhalt der Deiche und in Wach- und Polizeiaufgaben bestanden. Dabei waren die Dienste nach Stand und Vermögen unterschiedlich. Die Amtsträger der städtischen Magistratur und die für die Heeresversorgung zuständigen Epimeleten wurden aus der Mitte des Stadtrates bestimmt, ${ }^{2}$ die übrigen Stadtbewohner stellten in der Regel ihre eigene Arbeitskraft zur Verfügung. Besonders häufig belegt sind Aufgaben im Post- und Transportwesen sowie Wachdienste innerhalb der Stadt.

Die Organisation dieser einfachen, plebeischen Liturgien geschah durch die Phylen. Die neuen Liturgen wurden am Beginn des Jahres vom Leiter der dienstpflichtigen Phyle nominiert. Dabei wechselten sich die Phylen in einem jährlichen Turnus ab. In der Regel wurden alle in einem Jahr zu ernennenden Liturgen von einer Phyle gestellt. Wenn auch die Phylen die Aufgaben der vorhergehenden Organisation der Stadtbevölkerung in Vierteln, Amphoda, weiterführten, unterschieden sie sich jedoch in Ausdehnung und Anzahl. ${ }^{3}$ Ein erster Schritt zu einem besseren Verständnis der inneren Gliederung der Metropolen ist daher die Feststellung der jeweiligen Phylenzahl. Dank der Numerierung der Phylen und der zyklisch wiederkehrenden Dienstpflicht läßt sich das Schema bereits mit wenigen Daten nachzeichnen.

Aufgrund der für Oxyrhynchos vergleichsweise zahlreichen Belege konnten J. Rea und A. Bowman die Phylenzyklen dort für die 65 Jahre von der Einführung im Jahre 206 bis 271 rekonstruieren. ${ }^{4}$ Die späteren Belege fügen sich jedoch nicht in dieses Schema, so daß die Anzahl der Phylen in Oxyrhynchos in der Zeit ab Diokletian bisher nicht festgestellt werden konnte. Das 4. Jahrhundert ist für die Phylenzyklen in anderen Metropolen am besten dokumentiert. Aus dieser Zeit gibt es einzelne Belege von Phylen für Arsinoiton Polis, ${ }^{5}$ Panopolis und die Große Oase. ${ }^{6}$

\footnotetext{
${ }^{2} \mathrm{Zu}$ den Epimeleten F. Mitthoff, Annona I, S. 96-98.

${ }^{3}$ Für Oxyrhynchos ist mit J. Krüger, Oxyrhynchus in der Kaiserzeit, Frankfurt/Main 1990 (Europäische Hochschulschriften 441), S. 93f. rein rechnerisch von durchschnittlich zwei Amphoda pro Phyle auszugehen, doch gibt es auch Belege für wenigstens drei Amphoda in einer Phyle.

${ }^{4}$ A. Bowman, The Town Councils of Roman Egypt, Toronto 1971 (American Studies in Papyrology 11), S. 149-15, und P.Oxy XLIII, S. 23f.

${ }^{5}$ P.Vind. Tandem 4 von 313-315 n.Chr.

${ }^{6}$ Die 1. Phyle in P.Panop. 19, Kol. V, 3 von 342 n.Chr. Ein Liturgievorschlag aus dem Jahre 369/370, SB XVIII 13252. Das späte Auftreten des Amtes eines Phylarchen erklärt die Erstherausgeberin mit der Abgeschiedenheit des Ortes, B. Kramer, Zwei Leipziger Papyri, APF 32, 1986, 33-46, S. 41.
} 
Dazu kommen mehrere Belege aus Oxyrhynchos und aus Hermupolis, wo die Dokumentation bis ins 6. Jahrhundert reicht.

Im Folgenden soll der Versuch einer Rekonstruktion der Phylenzahl und des Phylenzyklus in der zweiten Hälfte des 4. Jahrhunderts für Hermupolis und Oxyrhynchos unternommen werden. Diese Rekonstruktion erlaubt zum ersten Mal einen Vergleich zwischen zwei ägyptischen Metropolen.

Um die Prinzipien der neuen Rekonstruktion zu bestimmen, lohnt sich ein Blick auf den bereits bekannten Zeitraum in Oxyrhynchos, wie er von J. Rea und A. Bowman rekonstruiert wurde. ${ }^{7}$ Der Dienst begann jeweils am ersten Thoth des betreffenden Jahres, also dem 29. August bzw. bei folgendem Schaltjahr dem 30. August, und dauerte dann bis zum 28. bzw. 29. August des Folgejahres. Der Übersichtlichkeit halber ist im Folgenden jeweils nur die Jahreszahl des Beginns der Liturgie eingetragen. Die Zahlen sind also so zu lesen, daß die erste Phyle am 29. August $206 \mathrm{ihr}$ Dienstjahr der Liturgieleistungen begann, das dann regulär bis zum 28. August des Folgejahres dauerte. Nach diesem Schema wurden insgesamt vier vollständige Zyklen durchgeführt (206-229 n.Chr.). Die Situation ändert sich mit dem Jahr 230, von dem an - vermutlich aufgrund von Schwierigkeiten, genügend Liturgen zu finden - jeweils zwei Phylen in einem Jahr zusammen Dienst tun. ${ }^{8}$ Die größere Anzahl an potentiellen Liturgen ist freilich dadurch erkauft, daß die einzelnen Phylen häufiger in die Pflicht genommen werden. Während der 15 Jahre, in denen dieses Schema durchgeführt wurde (230-244 n.Chr.) war jede Phyle fünfmal dienstpflichtig, in den vorhergehenden 24 Jahren nur viermal. Das entgegengesetzte Prinzip gilt in den Jahren ab Philippus Arabs, in denen eine Zahl von zwölf Phylen belegt ist. ${ }^{9}$ Anscheinend wurden die bisherigen Phylen halbiert und neu gezählt, so daß die frühere 1 . Phyle nun in eine neue Phyle 1 und eine neue Phyle 2, die frühere zweite Phyle in eine neue Phyle 3 und eine neue Phyle 4 usw. aufgeteilt wurde. Der letzte Beleg gilt

\footnotetext{
${ }^{7}$ Für das Folgende vgl. die Übersichten und Darstellungen bei J. Krüger, Oxyrhynchus in der Kaiserzeit (s.o. Anm. 3), S. 91f., und U. Kunnert, Bürger unter sich (s.o. Anm. 1), S. 211.

${ }^{8}$ So J. Rea zu P.Oxy. XLIII 3095-3098.

${ }^{9} \mathrm{Zu}$ den Reformen vgl. P.J. Parsons, Philippus Arabs and Egypt, JRS 57, 1967, 134 141.
} 
der 3. Phyle als dienstpflichtig für das Jahr 271. Danach klafft in der Überlieferung eine Lücke von 80 Jahren. ${ }^{10}$

1) Oxyrhynchos 206-229 n.Chr.: sechs Phylen im Jahreswechsel

$\begin{array}{lllll} & \text { Zyklus 1 } & \text { Zyklus 2 } & \text { Zyklus 3 } & \text { Zyklus } \\ \text { Phyle 1 } & 206 & 212 & 218 & 224 \\ \text { Phyle 2 } & 207 & 213 & 219 & 225 \\ \text { Phyle 3 } & 208 & 214 & 220 & 226 \\ \text { Phyle 4 } & 209 & 215 & 221 & 227 \\ \text { Phyle 5 } & 210 & 216 & 222 & 228 \\ \text { Phyle 6 } & 211 & 217 & 223 & 229\end{array}$

2) Oxyrhynchos 230-244 n.Chr.: drei Doppelphylen im Jahreswechsel

$\begin{array}{llllll} & \text { Zyklus 1 } & \text { Zyklus 2 } & \text { Zyklus 3 } & \text { Zyklus 4 } & \text { Zyklus 5 } \\ \text { Phylen 1+2 } & 230 & 233 & 236 & 239 & 242 \\ \text { Phylen 3+4 } & 231 & 234 & 237 & 240 & 243 \\ \text { Phylen 5+6 } & 232 & 235 & 238 & 241 & 244\end{array}$

3) Oxyrhynchos 245-271 n.Chr.: zwölf Phylen im Jahreswechsel

$\begin{array}{llll} & \text { Zyklus 1 } & \text { Zyklus 2 } & \text { Zyklus 3 } \\ \text { Phyle 1 } & 245 & 257 & 269 \\ \text { Phyle 2 } & 246 & 258 & 270 \\ \text { Phyle 3 } & 247 & 259 & 271 \\ \text { Phyle 4 } & 248 & 260 & \\ \text { Phyle 5 } & 249 & 261 & \\ \text { Phyle 6 } & 250 & 262 & \\ \text { Phyle 7 } & \mathbf{2 5 1} & \mathbf{2 6 3} & \end{array}$

\footnotetext{
${ }^{10}$ Der Hinweis auf die 2. Phyle in P.Oxy. XIV 1652 (314-315), Z. 43, steht in zerstörtem Zusammenhang, so daß keine Aussage darüber möglich ist, ob sie damals liturgiepflichtig war.
} 


$\begin{array}{lcc}\text { Phyle 8 } & 252 & 264 \\ \text { Phyle 9 } & 253 & 265 \\ \text { Phyle 10 } & 254 & 266 \\ \text { Phyle 11 } & 255 & 267 \\ \text { Phyle 12 } & 256 & 268\end{array}$

Aus den vorliegenden Daten lassen sich einige Eigenschaften dieses Systems zur Bestimmung der Phylenzyklen in späterer Zeit ableiten:

1. Solange die Phylen durchnumeriert sind, liegt beim jährlichen Wechsel durch eine datierte Angabe der dienstpflichtigen Phyle gleichzeitig auch das erste Jahr des Zyklus fest. So läßt sich zum Beispiel aus der Angabe, daß im Jahr 215 die vierte Phyle dienstpflichtig war, der Beginn des Zyklus auf 212 festlegen. Voraussetzung ist jedoch, daß jedes Jahr nur eine Phyle dienstpflichtig ist. Diese Voraussetzung ist jeweils zu überprüfen; ohne Berücksichtigung des Doppelzyklus zwischen 230-244 würde man etwa aus Angabe der Dienstpflicht von Phyle 4 im Jahr 234 fälschlich auf das Jahr 231 als Beginn des Zyklus schließen.

2. Zur Bestimmung der Länge des Zyklus werden Angaben aus zwei aufeinander folgenden Zyklen benötigt. Die Länge des Zyklus und die Anzahl der Phylen ergibt sich, indem man die jeweiligen Dienstjahre derselben Phyle voneinander abzieht, am Beispiel der 3. Phyle 214 $-208=6$ Jahre und 6 Phylen. Sind nur Dienstjahre unterschiedlicher Phylen belegt, so sind die Angaben jeweils auf das Dienstjahr der ersten Phyle zurückzuführen, bevor die Differenz zwischen den Jahreszahlen gebildet werden kann, im Beispiel also auf 212-206=6.

3. Zusätzliche Vorsicht erfordert auch die Unvollständigkeit der Belege. Wären allein die Dienstjahre 215 und 227 für die vierte Phyle belegt, könnte man fälschlicherweise auf einen Zyklus und eine Phylenanzahl von 12 schließen. ${ }^{11}$ Zum Gegenbeweis wäre hier ein Beleg aus dem ersten oder dritten Zyklus erforderlich. Sonst müssen jeweils mehrere Möglichkeiten in Betracht gezogen werden.

\footnotetext{
${ }^{11}$ Allerdings wurde zu Beginn noch die laufende Nummer des Zyklus angegeben, z.B.

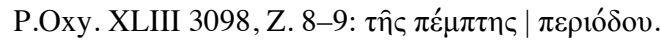


Für das 4. Jahrhundert sind liturgiepflichtige Phylen sowohl in Oxyrhynchos als auch und zum ersten Mal in Hermupolis belegt. Es ist möglich, daß sich die Anzahl der Phylen und die Häufigkeit ihrer Dienstpflicht in der Zwischenzeit immer wieder geändert haben. Einfacher ist die Rekonstruktion in Hermupolis, weil dort die Phylen wie im Oxyrhynchos des 3. Jahrhunderts numeriert sind. Allerdings ergeben sich rechnerisch zwei Möglichkeiten, weil von den drei Belegen nur zwei genau datiert sind. Der Übersichtlichkeit halber stelle ich das Ergebnis der Rekonstruktion an den Anfang. Die belegten Daten sind darin unterstrichen:

\section{Hermupolis 354-397 n.Chr.: 16 Phylen im Jahreswechsel}

\section{Zyklus 2. Zyklus 3. Zyklus}

$\begin{array}{llll}\text { Phyle 1 } & 354 & 370 & 386 \\ \text { Phyle 2 } & 355 & 371 & 387 \\ \text { Phyle 3 } & 356 & 372 & 388 \\ \text { Phyle 4 } & 357 & 373 & 389 \\ \text { Phyle 5 } & 358 & 374 & \underline{390} \\ \text { Phyle 6 } & 359 & 375 & 391 \\ \text { Phyle 7 } & 360 & 376 & 392 \\ \text { Phyle 8 } & 361 & 377 & 393 \\ \text { Phyle 9 } & 362 & 378 & 394 \\ \text { Phyle 10 } & 363 & 379 & 395 \\ \text { Phyle 11 } & 364 & 380 & 396 \\ \text { Phyle 12 } & 365 & 381 & \underline{397} \\ \text { Phyle 13 } & 366 & 382 & \\ \text { Phyle 14 } & 367 & 383 & \\ \text { Phyle 15 } & 368 & 384 & \\ \text { Phyle 16 } & \underline{369} & 385 & \end{array}$

Die Belege aus Hermupolis weisen alle kein Tagesdatum auf. Sie behandeln aber jeweils Ereignisse zu Beginn des Dienstjahres der Liturgen, wie die Präsentation von liturgiepflichtigen Personen oder Bürgschaften über die Erfüllung der Dienstpflicht von Liturgen. Daher ist anzunehmen, daß die Urkunden, ebenso wie die vergleichbaren Papyri aus Oxyrhynchos, jeweils um den 29. August ausgestellt wurden. 
Der erste Beleg, P.Lips. I 65, wurde bereits 1902 von Ludwig Mitteis veröffentlicht $;{ }^{12}$ das Original ist heute verschollen. Es handelt sich um eine Präsentation von liturgiepflichtigen Personen an den Nyktostrategen Aurelius Kyros aus dem Jahre 390 n.Chr. ${ }^{13}$ Die Meldung erfolgt durch Aurelius Nilos, Sohn des Ammon, den Gnoster der 5. Phyle, welche dementsprechend als für dieses Jahr dienstpflichtig anzunehmen ist.

Dazu stimmt der Beleg aus P.Würzb. Inv. 77, einer an den Nyktostrategen Hyperechius gerichteten Gestellungsbürgschaft für einen Wächter aus dem Jahr 397, durch den wir erfahren, daß in diesem Jahr die 12. Phyle in Hermupolis dienstpflichtig war (Z. 11-12).${ }^{14}$ Aus diesen beiden Belegen läßt sich schließen, daß zu dieser Zeit in Hermupolis die Phylen sich einzeln in einem jährlichen Turnus abwechselten, der im Jahre 386 mit der 1. Phyle begonnen hatte.

Bei beiden Fassungen von PSI I 86 sind mit dem Anfang und Ende des Textes auch die Ortsangabe und die Konsulardatierung verloren gegangen. Die allgemein akzeptierte Zuweisung an den Hermopolites beruht auf einem Eigennamen und den verwendeten Formeln. ${ }^{15}$ Die Datierung erfolgt nach den Regierungszeiten der im Kaisereid genannten Herrscher Valentinian, Valens und Gratian (Z. 4-5) auf die Jahre 367-375 n.Chr. Es handelt sich um eine Bürgschaft für die Erfüllung einer einjährigen bürgerlichen

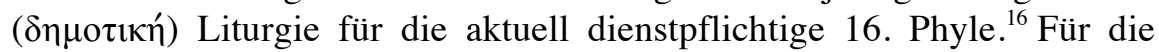

\footnotetext{
${ }^{12}$ L. Mitteis, Griechische Papyri zu Leipzig, APF 2, 1902, 259-272 (hier: 262-264).

${ }^{13}$ Der Adressat begegnet uns in einer ganzen Reihe von Papyri, die Daniela Colomo zu einem Dossier von bisher 33 Texten zusammengefaßt hat. Davon befinden sich 29 in Leipzig, die übrigen in Giessen und Straßburg. Überwiegend handelt es sich um die Meldung liturgiepflichtiger Personen zum Wachdienst aus dem Jahr 390, doch reicht die Dokumentation insgesamt bis 398. Vgl. R. Scholl/M. Homann, Gesucht und gefunden. Amtliche(?) Anweisung zur Unterlassung von Belästigung, APF 55, 2009, 462-469, S. 465.

${ }^{14}$ Descriptum bei H. Essler, Über die Würzburger Papyrussammlung, PapCongr. XXIV, S. 295.

${ }^{15}$ Vgl. N. Gonis, Notes on Miscellaneous Documents II, ZPE 154, 2005, 203-213, S. 209 Anm 19, und K.A. Worp, Byzantine Imperial Titulature in the Greek Documentary Papyri: The Oath Formulas, ZPE 45, 1982, S. 208.

${ }^{16}$ Die Angabe der in diesem Falle diensttuenden 16. Phyle ergibt eine ungewöhnlich hohe Phylenzahl, die öfter Anlaß zum Zweifel an der stets erneut bestätigten Lesung der Zahl gab, vgl. R.S. Bagnall/K.A. Worp, Notes on Byzantine Documents, IV, BASP 17,

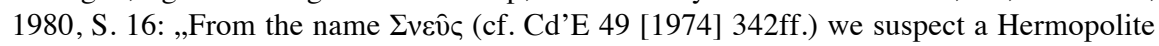
provenance for the papyrus, and we do not know enough about the tribal system there to be certain if the phrase makes sense." Vgl. die Reedition von P. Pruneti: Un atto di garanzia
} 
Anbindung dieses Belegs an den 386 beginnenden Zyklus der beiden anderen Belege gibt es rechnerisch elf Möglichkeiten: Die Phylennummer 16 aus PSI I 86 gibt den Mindestwert für die Länge eines Zyklus. Das erste Jahr dieses Zyklus liegt 15 Jahre früher, also zwischen $367-15=352$ und 375-15=360. Um den Abstand zwischen den Anfängen der beiden Zyklen zu bestimmen, werden die Jahreszahlen des Dienstjahres der jeweils ersten Phyle voneinander abgezogen. Der Abstand beträgt damit zwischen 386-352=34 und 386-360=26 Jahre. Theoretisch könnten die Belege aus zwei direkt aufeinanderfolgenden Zyklen stammen, jedoch wird man diese neun Möglichkeiten angesichts der unerhört großen Zyklenlänge von 26-34 Jahren und der hohen Zahl von 26-34 Phylen wohl ausschließen dürfen. Liegt ein vollständiger Zyklus zwischen den beiden Belegen, sind die Abstandswerte zu halbieren. Es ergibt sich damit eine rechnerische Länge des Zyklus von 13-17 Jahren und eine Anzahl von 13-17 Phylen. Da es nach PSI I 86 mindestens 16 Phylen gab, bleiben nur noch die beiden Möglichkeiten mit 16 Phylen (bei einer Datierung von PSI I 86 ins Jahr 369) und mit 17 Phylen (bei einer Datierung ins Jahr 367). Der Beginn dieses Zyklus fiele ins Jahr 354 beziehungsweise 352. ${ }^{17}$

Für eine Anzahl von 16 Phylen in Hermupolis spricht neben der praktischen Überlegung der einfacheren weiteren Untergliederung oder $\mathrm{Zu}$ sammenlegung der Phylen wohl auch die unpublizierte Quittung P.Lips. Inv. 1115. In diesem paläographisch auf das vierte Jahrhundert datierten Papyrus wird ein Zyklus von insgesamt 16 Phylen einer nicht näher ge-

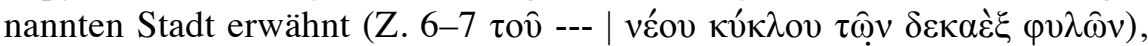
bei der es sich wohl, wie bei P.Lips. I 65, um Hermupolis handeln dürfte. ${ }^{18}$ Leider läßt sich dieses Schema nicht bis zum nächsten Beleg, CPR XXIII 33 (Hermupolis, 12.11.550), fortführen. Dort ist eine aktuell diensttuende 8. $\pi \alpha \gamma \alpha v ı$ ń Phyle genannt (Z. 4). ${ }^{19}$ Die Fortführung unseres rekonstruierten Phylenzyklus ergäbe im Jahr 550 als dienstpflichtig entweder die 5.

del IV secolo: PSI I 86, in: Studi in onore di Arnaldo Biscardi I (Milano 1982), S. 369-377.

${ }^{17}$ Für die Zeit davor kenne ich keine Belege, doch wird man wie in Oxyrhynchos auch in Hermupolis des 3. Jahrhunderts mit Phylen zu rechnen haben.

${ }^{18} \mathrm{Vgl}$. den Katalogeintrag von D. Colomo unter <http://papyri.uni-leipzig.de/receive/ UBLPapyri_schrift_00006210>, zuletzt aufgerufen am 23.4.2016.

${ }^{19}$ F. Mitthoff, CPR XXIII, S. 203-206, vermutet, es handle sich um eine plebeische städtische Phyle; dagegen U. Kunnert: Bürger unter sich (s.o. Anm. 1), S. 215; die Belege für Dorfphylen ebd. Anm. 857. Zu den gelegentlichen Beiträgen von Funktionären aus den Dörfern zum Wachdienst in der Gaumetropole vgl. D. Hennig, Nyktophylakes, Nykto-

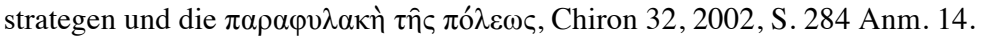


Phyle (im Falle von 16 Phylen) oder die 12. Phyle (im Falle von 17 Phylen).

In Oxyrhynchos sind zwischen dem 3. und 4. Jahrhundert mehrere Änderungen eingetreten. Sie finden ihren Niederschlag in neuen Bezeichnungen der Phylen selbst und der für ihre Leitung verantwortlichen Beamten. Im Laufe des 3. Jahrhunderts löst um 245 der $\varphi v ́ \lambda \alpha \rho \chi o \varsigma$ den $\alpha \mu \varphi$ $\mu \delta о \gamma \rho \alpha \mu-$ $\mu \alpha \tau \varepsilon v ́ c$ als Vorsteher der Phylen ab, um in den 280er Jahren wiederum

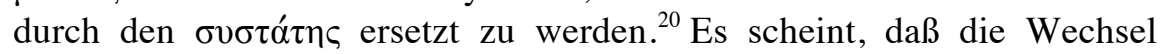
jeweils mit einem neuen Zyklus eintraten. An die Stelle der Numerierung des 3. Jahrhunderts ist ab Mitte des 4. Jahrhunderts die Benennung der Phylen nach Stadtteilen getreten. Für die Rekonstruktion des Zyklus ergibt sich daraus die Schwierigkeit, daß die Stelle der dienstpflichtigen Phyle innerhalb der Reihenfolge nicht mehr aus ihrem Namen abgeleitet werden kann. Mit Abstand am häufigsten belegt ist die Phyle um den Gymnasion-

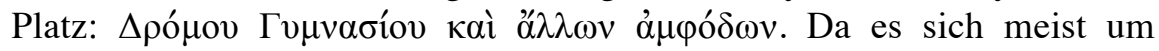
Nominierungen von Liturgen handelt, fallen die Daten der Belege um den 1. Thoth (29./30. August), den Beginn des Dienstjahres der Liturgen. Die meisten Belege rühren von Aurelius Muses, der wenigstens 30 Jahre (356-386) als Systates dieser Phyle amtierte, und seinem Sohn Aurelius Heliodoros her. Insgesamt gibt es acht Belege dieser Phyle aus den Jahren 356-412, die sich aber nicht alle in ein einheitliches Schema bringen lassen. So liegen etwa zwischen dem ersten und zweiten Beleg drei, zwischen dem zweiten und dritten Beleg vier Jahre. Allerdings ist nur in drei Fällen unsere Phyle explizit als gerade dienstpflichtig genannt, indem

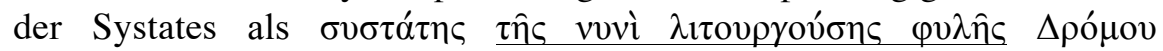

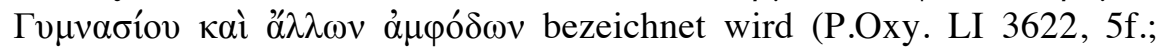
P.Coles 28, 5f.; P.Oxy. LV 3796, 4) ${ }^{21}$ Sie sind in der folgenden Aufstellung durch Unterstreichung hervorgehoben. In allen anderen Fällen ist

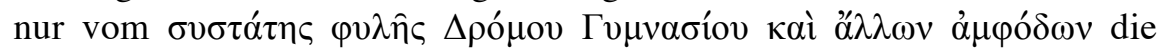
Rede (P.Oxy. LI 3623, 5; P.Oxy. VIII 1116, 5; PSI X 1108, 5; P.Oxy.

\footnotetext{
${ }^{20}$ Ursula Kunnert: Bürger unter sich (s.o. Anm. 1), S. 212, Anm. 847. Zum Wechsel der Bezeichnung vgl. auch F. Abou Bakr, The Office of the Phylarch at Oxyrhynchus, BSAA 46, 2000, 1-6, S. 1f. und 5f.

${ }^{21}$ F. Abou Bakr, The Office of the Phylarch at Oxyrhynchus, BSAA 46, 2000, 1-6, S. 4 Anm. 46, sammelt Belege aus den Jahren 269-270, in denen der ehemalige Phylarch ( $\varphi v ́ \lambda \alpha \rho \chi \circ \varsigma$ $\gamma \varepsilon v o ́ \mu \varepsilon v \circ \varsigma$ ) weiterhin für die Bürgerlisten der Getreideausgabe verantwortlich zeichnet, obwohl seine Phyle gerade nicht dienstpflichtig ist und daher keinen amtierenden Phylarchen hat: P.Oxy. XL 2892, 2895, 2896 und 2907.
} 
XXXIV 2715, 5; P.Oxy. LXXII 4898, 3), wobei oft der Liturge als aus der

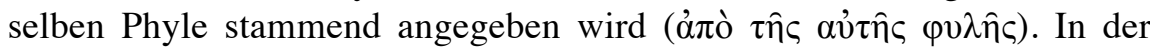

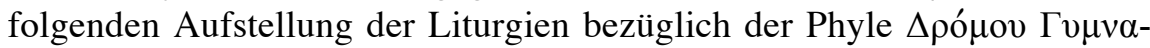

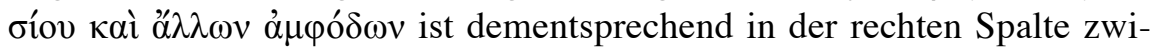
schen diesen beiden Angaben über die Dienstpflicht der Phyle und der Herkunft des Liturgen unterschieden. Fehlen beide Angaben, ist der Eintrag leer gelassen.

\begin{tabular}{|c|c|c|c|c|c|}
\hline Jahr & Tag & Papyrus & Inhalt & Systates & $\begin{array}{l}\text { Bezug } \\
\text { zur Phyle }\end{array}$ \\
\hline 356 & 29.8. & P.Oxy. LI 3622 & $\begin{array}{l}\text { Übernahme } \\
\text { einer Liturgie }\end{array}$ & Aur. Muses & $\begin{array}{l}\text { aktuell } \\
\text { liturgie- } \\
\text { pflichtig }\end{array}$ \\
\hline 359 & & P.Oxy. LI 3623 & $\begin{array}{l}\text { Liturgievor- } \\
\text { schlag }\end{array}$ & Aur. Muses & \\
\hline 363 & $\begin{array}{l}30.8 .- \\
28.9 \text {. }\end{array}$ & P.Oxy. VIII 1116 & $\begin{array}{l}\text { Liturgievor- } \\
\text { schlag } \\
\text { Alexandria }\end{array}$ & Aur. Muses & $\begin{array}{l}\text { Liturge } \\
\text { aus der } \\
\text { selben } \\
\text { Phyle }\end{array}$ \\
\hline 381 & 2.9 .22 & PSI X 1108 & $\begin{array}{l}\text { Liturgievor- } \\
\text { schlag }\end{array}$ & Aur. Muses & $\begin{array}{l}\text { Liturge } \\
\text { aus der } \\
\text { selben } \\
\text { Phyle }\end{array}$ \\
\hline $\begin{array}{l}384 \\
\text { oder } \\
385\end{array}$ & 29.8. & P.Coles 28 & $\begin{array}{l}\text { Liturgievor- } \\
\text { schlag } \\
\text { Post }\end{array}$ & $\begin{array}{l}\text { Aur. } \\
\text { Heliodoros }\end{array}$ & $\begin{array}{l}\text { aktuell } \\
\text { liturgie- } \\
\text { pflichtig }\end{array}$ \\
\hline 385 & 29.8. & $\begin{array}{l}\text { P.Oxy. XXXIV } \\
2715\end{array}$ & $\begin{array}{l}\text { Liturgievor- } \\
\text { schlag }\end{array}$ & Aur. Muses & $\begin{array}{l}\text { Liturge } \\
\text { aus der } \\
\text { selben } \\
\text { Phyle }\end{array}$ \\
\hline 391 & & $\begin{array}{l}\text { P.Oxy. LXXII } \\
4898\end{array}$ & $\begin{array}{l}\text { nur Erwähnung } \\
\text { des Systates }\end{array}$ & $\begin{array}{l}\text { Aur. } \\
\text { Heliodoros }\end{array}$ & \\
\hline
\end{tabular}

\footnotetext{
${ }^{22}$ Da der in PSI X 1108 angegebene Tag, der 5. Thoth, als Datum für die Nominierung des Liturgen nach dem Dienstantritt zum 1. Thoth liegt, verbesserte der Herausgeber das Epsilon zu Alpha. Dagegen wandten sich P.J. Sijpesteijn und K.A. Worp, Chronological Notes, ZPE 26, 1977, 267-286, S. 274: „We see no reason why we should not take the PSI papyrus at face value and date the papyrus to 2.9.381 A.D. Although the nominee had to start his liturgy on the 1 st of Thoth, his nomination was proposed only on the $5^{\text {th }}$ of Thoth."
} 
Übernahme einer Liturgie aktuell liturgiepflichtig

Betrachten wir zunächst nur die drei Belege, in denen die Phyle explizit als liturgiepflichtig bezeichnet wird. Leider ist das Jahr des mittleren Beleges (P.Coles 28) unsicher. Die Konsulardatierung beginnt mit $i \pi \alpha \tau \varepsilon i ́ \alpha v$, welches entweder in $v \pi \alpha \tau \varepsilon^{\prime} \alpha \varsigma$ (mit einer Datierung auf 384) zu korrigieren

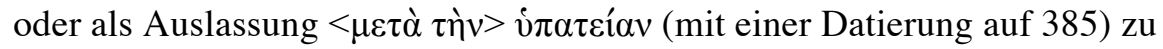
verstehen ist. ${ }^{23}$ Nimmt man den 29.8.384 als Datum für diesen Papyrus an, beträgt der Abstand zum vorhergehenden Zeugnis (P.Oxy. LI 3622, 29.8.356) und zum folgenden (P.Oxy. LV 3796, 10.12. 412) jeweils 28 Jahre, während die Annahme einer Datierung auf den 29.8.385 zu Abständen von 29 und 27 Jahren führt. Ich gehe daher von der ersten Möglichkeit aus, zumal die Primzahl 29 eine Rekonstruktion unmöglich machen würde. Während des zum 1. Thoth 385 beginnenden Liturgiejahres ist außerdem - in unklarem Zusammenhang - der Systates einer anderen Phyle

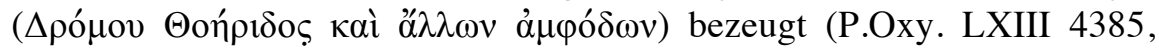
22.5.386) ${ }^{24}$ Genannt sind in dem Vertrag Angehörige jener Phyle, möglicherweise im Hinblick auf ihre Dienstpflicht. ${ }^{25}$ Ferner würde bereits im darauffolgenden Dienstjahr erneut ein Mitglied der Phyle um den Gymnasion-Platz zur Dienstleistung auf den staatlichen Schiffen nominiert (P.Oxy. XXXIV 2715, 29.8.386). Bei der Datierung von P.Coles 28 auf das Jahr 384 sind rechnerisch Zyklenlängen von 2, 4, 7, 14 und 28 Jahren möglich.

Nicht alle der übrigen Belege können in einen einzigen Zyklus dieser Länge eingeordnet werden. Es ist entweder davon auszugehen, daß einige dieser Urkunden in Jahren entstanden sind, in denen unsere Phyle nicht als ganzes liturgiepflichtig war und die belegten Liturgen außerhalb des regu-

${ }^{23}$ Der ersten Datierung wird bei N. Gonis zu P.Oxy. LXXII 4898, Anm. zu 2-3, der zweiten bei N. Gonis zu P.Coles Anm. zu 1-2 mit Hinweis auf den immer noch nach dem Postkonsulat des vorhergehenden Jahres datierten P.Oxy. LXIII 4383 vom 24.10.384 der Vorzug gegeben. Ich danke N. Gonis sehr herzlich für die Mitteilung seiner im Druck befindlichen Edition dieses Papyrus.

${ }^{24}$ P.Oxy. LXIII 4385 selbst stammt aus der Zeit nach dem Datum des Vertrages (22.5.386), weil es sich um eine Abschrift davon handelt. Vielleicht ist ein Bezug auf die Liturgien des Ende August beginnenden Dienstjahres in $\chi \rho o ́ v \omega$ in Z. 7 zu sehen.

${ }^{25}$ Es handelt sich um einen Vertrag zwischen zwei Parteien unklaren Inhaltes, den J. Rea in der Einleitung zur Edition, P.Oxy. LXIII, S. 94, jedoch vermutungsweise in Zusammenhang mit Liturgien bringt. 
lären Dienstjahres nominiert wurden, oder daß unsere Phyle in unregelmäßigen Abständen dienstpflichtig war.

Nach der Verteilung der übrigen Zeugnisse ist am wahrscheinlichsten eine Zyklenlänge von 7 Jahren, zu der zwei Belege stimmen, in denen der

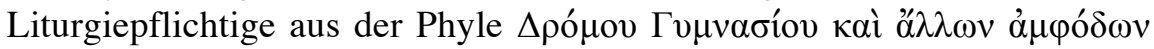
stammt: In P.Oxy. VIII 1116 wird das nächste Mitglied der Phyle von der Liturgenliste für den Dienst am Augusteum in Alexandria nominiert. Die erhaltene Datierung lautet auf Thoth des Jahres 363. Die weggebrochene Tagesangabe ist vermutlich auf den 30. August (1. Thoth, Z. $2 \Theta \dot{\omega}[\theta \alpha]$ ) zu ergänzen, zum einen wegen der übrigen Parallelen, die alle zum Beginn des Liturgiejahres datieren, zum anderen, weil die Liturgie am 1. Thoth beginnen soll (Z. 12f.). P.Oxy. LXXII 4898 (391) enthält nur den Anfang einer Urkunde mit der Konsulardatierung und der Nennung des Systates unserer Phyle, Aurelius Heliodoros, Sohn des Muses. ${ }^{26}$

Schwer zu vereinbaren mit einem Zyklus eines Umfanges, der mit den oben genannten Werten kompatibel ist, sind die beiden auf Postdienste bezogenen Liturgien in P.Oxy. LI 3623 (359) und PSI X 1108 (2.9.381), die 22 Jahre auseinander und von unseren sicheren Zeugnissen 3 bzw. 5 Jahre entfernt liegen. In P.Oxy. LI 3623 handelt es sich um eine Nominierung durch den Systates unserer Phyle, jedoch sind keine Angaben über die aktuell dienstpflichtige Phyle und die Phylenzugehörigkeit des dienstpflichtigen Liturgen erhalten. In PSI X 1108 gehört der Liturgiepflichtige zu unserer Phyle. Dazu kommt der bereits erwähnte P.Oxy. XXXIV 2715, für die Liturgie auf den staatlichen Schiffen. Es ist möglich, daß diese überregionalen Transport- und Postaufgaben nach einem anderen Schema vergeben wurden als der Liturgiezyklus in der Metropole. Die Amtszeit scheint jedoch ebenfalls ein Jahr betragen zu haben.

Muß die Frage nach der genauen Anzahl der Phylen und der Länge des Zyklus für Oxyrhynchos offenbleiben, so läßt sich jedenfalls feststellen, daß dort im 4. Jahrhundert die Länge des Zyklus und damit wohl auch die Phylenzahl sowohl inkommensurabel zu den 6 bzw. 12 Phylen des 3. Jahrhunderts als auch zu den 16 in Hermupolis belegten Phylen ist. ${ }^{27}$

\footnotetext{
${ }^{26}$ Für die Möglichkeit, daß es sich beim Adressaten um den Sohn von Aurelius Muses handelt, vgl. den Kommentar von N. Gonis zu P.Oxy. 4898, Z. 2-3.

${ }^{27}$ Das selbe gilt für den Zyklus von fünf Phylen, den E.P. Wegener, Notes on the $\varphi v \lambda \alpha$ í of the metropoleis, PapCongr. V, S. 513-518, allerdings für das 3. Jh. angenommen hatte. Dagegen zu Recht $\mathrm{P}$. Mertens, Les services de l'état civil et le contrôle de la population à Oxyrhynchus au III ${ }^{e}$ siècle de notre ère, Bruxelles 1968, S. 45 Anm. 234.
} 